\title{
MAGNETO CONVECTION IN A LAYER OF NANOFLUID WITH SORET EFFECT
}

\author{
Ramesh CHAND*, Gian Chand RANA** \\ *Department of Mathematics, Government Arya Degree College Nurpur, Himachal Pradesh, 176202, India \\ *Department of Mathematics, Government College Nadaun, Himachal Pradesh, 177103, India \\ rameshnahan@yahoo.com, drgcrana15@gmail.com
}

received 22 February 2015, revised 22 July 2015, accepted 24 July 2015

\begin{abstract}
Double diffusive convection in a horizontal layer of nanofluid in the presence of uniform vertical magnetic field with Soret effect is investigated for more realistic boundary conditions. The flux of volume fraction of nanoparticles is taken to be zero on the isothermal boundaries. The normal mode method is used to find linear stability analysis for the fluid layer. Oscillatory convection is ruled out because of the absence of the two opposing buoyancy forces. Graphs have been plotted to find the effects of various parameters on the stationary convection and it is found that magnetic field, solutal Rayleigh number and nanofluid Lewis number stabilizes fluid layer, while Soret effect, Lewis number, modified diffusivity ratio and nanoparticle Rayleigh number destabilize the fluid layer.
\end{abstract}

Keywords: Nanofluid, Zero-Flux, Soret Effect, Nanofluid Lewis Number, Chandrasekhar Number, Magnetic Field, Galerkin Method

\section{INTRODUCTION}

Double-diffusive convection is referred to convection induced by temperature and concentration gradients or by concentration gradients of two spices. Double diffusive convection has become important in recent years because of its applications in many fields of science, engineering and technology. Nield (1968) investigate the double-diffusive convection using linear stability analysis for various thermal and solutal boundary conditions. The onset of the double-diffusive convection by using a weak nonlinear theory was investigated by Rudraiah et al.(1982). Later Nield et al. (1993) considered the effects of inclined temperature and solutal gradients on the double convection. Thermal convection in binary fluid driven by the Soret and Dufour effect has been investigated by Knobloch (1980).He has shown that the equations are identical to the thermosolutal problem except relation between the thermal and solutal Rayleigh numbers. Bahloulet al. (2003) investigated the effects of Soret (thermal diffusion) in double -diffusive flow. Thermosolutal convection in the presence of Dufour and Soret effects by Motsa (2008) and found that Soret parameter stabilize while Dufour parameter destabilize the stationary convection. Dufour and Soret effects on thermosolutal convection in a viscoelastic fluid layer have been given by Chand and Rana (2012a, 2014a), Chand et al. (2015a)and observed that Dufour and Sore parameters have significance influence on the fluid layer.

The effect of magnetic field on double-diffusive convection finds importance in geophysics, particularly in the study of Earth's core where the Earth's mantle, which consists of conducting fluid. Magnetic field plays an important role in engineering and industrial applications. These applications include design of chemical processing equipment, formation and dispersion of fog, distributions of temperature and moisture over agricultural fields and groves of fruit trees and damage of crops due to freezing and pollution of the environment etc. Chandrasekhar (1961) studied in detail the thermal convection in a hydromagnetics. Patil and Rudraiah
(1973), Alchaar et al.(1995) considered the problem of thermosolutal convection in the presence of magnetic field for different boundary conditions.

Nanofluids have novel properties that make them potentially useful in wide range of engineering applications where cooling is of primary concern. Nanofluid used as heat transfer, chemical nanofluids, smart fluids, bio-nanofluids, medical nanofluids (drug delivery and functional tissue cell interaction) etc. in many industrial applications. The term nanofluid refers to a fluid containing a suspension of nanoscale particles. This type of fluid is a mixture of a regular fluid, with a very small amount of suspended metallic or metallic oxide nanoparticles or nanotubes, which was first coined by Choi (1995). Suspensions of nanoparticles are being developed medical applications including cancer therapy. Convection in nanofluid based on Buongiorno's model [Buongiorno, (2006)] was studied by Tzou (2008a,b), Alloui et al.(2010), Kuznetsov and Nield (2010a, b , 2011), Nield and Kuznetsov (2009, 2010a, b,c, 2011), Kim et al. (2011), Chand and Rana (2012b,c,d), Chand et al. (2015b). Magneto- convection in a layer of nanofluid finds its applications in biomedical engineering such as MRI, plethora of engineering, power plant cooling systems as well as in computers. Yadav et al. (2013), Chand (2013), Gupta et al. (2013) and Chand and Rana (2014b, 2015) reported various application of magnetic field in a layer of nanofluid heated from below. But the choice of the boundary conditions imposed by them on nanoparticles fraction is somewhat arbitrary; it could be argued that zero-flux for nanoparticles volume fraction is more realistic. Recently Nield and Kuznetsov (2014), Chand el al. (2014), Chand and Rana $(2014 c, 2015)$ studied the thermal instability of nanofluid by taking normal component of the nanoparticle flux zero at boundary which is more physically realistic. Zero-flux for nanoparticles mean one could control the value of the nanoparticles fraction at the boundary in the same way as the temperature there could be controlled. In this paper an attempt has been made to study the magneto- convection in a horizontal layer 
of nanofluid with Soret effect for more realistic boundary conditions.

\section{MATHEMATICAL FORMULATIONS OF THE PROBLEM}

Consider an infinite horizontal layer of nanofluid of thickness $d$ bounded by surfaces $z=0$ and $z=d$ heated and soluted from below such that a constant temperature and concentration distribution is prescribed at the boundaries of the fluid layer. Fluid layer is acted upon by gravity force $g(0,0,-g)$ and a uniform vertical magnetic field $H(0,0, H)$ as shown in Fig. 1 . The temperature and concentration are taken to be $T_{0}$ and $C_{0}$ at $z=$ 0 and $T_{1}$ and $C_{1}$ at $z=d,\left(T_{0}>T_{1}, C_{0}>C_{1}\right)$. The reference scale for temperature and nanoparticles fraction is taken to be $T_{1}$ and $\varphi_{0}$ respectively.

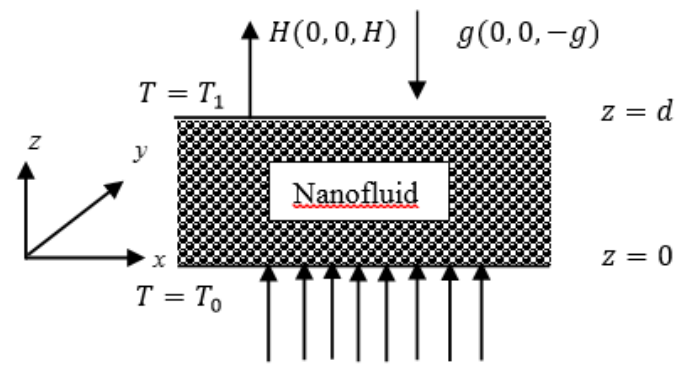

Heated from below

Fig.1. Physical configuration of the problem

\subsection{Assumptions}

The mathematical equations describing the physical model are based upon the following assumptions:

- Thermo physical properties expect for density in the buoyancy force (Boussinesq Hypothesis)are constant;

- The fluid phase and nanoparticles are in thermal equilibrium state;

- Nanoparticles are spherical;

- Radiation heat transfer between the sides of wall is negligible when compared with other modes of the heat transfer;

- Nanoparticles do not affect the transport of the solute;

- No chemical reactions take place in fluid layer.

\subsection{Governing Equations}

The appropriate governing equations for magneto double diffusive convection (Chandrasekhar, 1961; Chand, 2013;Nield and Kuznetsov, 2014) are:

$\nabla \cdot q=0$

$\rho_{0} \frac{\partial q}{\partial t}=-\nabla p+\left(\phi \rho_{p}+(1-\phi)\right.$

$\left.\cdot\left\{\rho_{0}\left(1-\alpha\left(T-T_{0}\right)+\alpha^{\prime}\left(C-C_{0}\right)\right)\right\}\right) g$ $\rho c \frac{\partial T}{\partial t}+(\rho c)_{f} q \cdot \nabla T=k_{m} \nabla^{2} T+\varepsilon(\rho c)_{p}$

$\cdot\left(D_{B} \nabla \phi \cdot \nabla T+\frac{D_{T}}{T_{1}} \nabla T \cdot \nabla T\right)$

$\frac{\partial C}{\partial t}+q \cdot \nabla C=\kappa \nabla^{2} C+D_{C T} \nabla^{2} T$

$\frac{\partial \phi}{\partial t}+q \cdot \nabla \phi=D_{B} \nabla^{2} \phi+\frac{D_{T}}{T_{1}} \nabla^{2} T$

where: $q(u, v, w)$ is the velocity vector, $\rho_{0}$ is the density of nanofluid at lower boundary layer, $\varphi$ is the volume fractionof the nanoparticles, $\rho_{p}$ density of nanoparticles, $p$ is the hydrostatic pressure, $\mu$ is the velocity viscosity, $\mu_{e}$ is the magnetic permeability, $\alpha$ is thecoefficientof thermal expansion, $\alpha^{\prime}$ an analogous solvent coefficient of expansion, $\kappa$ is the thermal diffusivity, $\kappa^{\prime}$ is the solute diffusivity of fluid, $H$ is the magnetic field, $T$ is the temperature, $C$ is the solute concentration, $\rho c$ is the effective heat capacity of fluid, $(\rho c)_{p}$ is the heat capacity of nanoparticles, $k_{m}$ is the effective thermal conductivity of the porous medium, $g$ is acceleration due to gravity, $D_{B}$ is the Brownian diffusion coefficient, $D_{T}$ is thethermophoretic diffusion coefficient of the nanoparticles and $D_{C T}$ is the Soret coefficient.

Maxwell equations are:

$\frac{d H}{d t}=(H \cdot \nabla) q+\eta \nabla^{2} H$

$\nabla \cdot H=0$

where $\eta$ is the electrical resitivity.

We assume that the temperature is constant and nanoparticles flux is zero on the boundaries. Thus boundary conditions (Chandrasekhar, 1961; Nield and Kuznetsov, 2014) are:

$w=0, T=T_{0,}, C=C_{0}, D_{B} \frac{\partial \varphi}{\partial z}+\frac{D_{T}}{T_{1}} \frac{\partial T}{\partial z}=0 a t z=0$

$w=0, T=T_{1}, C=C_{1}, D_{B} \frac{\partial \varphi}{\partial z}+\frac{D_{T}}{T_{1}} \frac{\partial T}{\partial z}=0 a t z=d$

Introducing non-dimensional variables as:

$\left(x^{\prime}, y^{\prime}, z^{\prime}\right)=\left(\frac{x, y, z}{d}\right), q^{\prime}\left(u^{\prime}, v^{\prime}, w^{\prime}\right)=q\left(\frac{u, v, w}{\kappa}\right) d$, $t^{\prime}=\frac{\kappa}{d^{2}} t, p^{\prime}=\frac{d^{2}}{\mu \kappa} p, \phi^{\prime}=\frac{\left(\phi-\phi_{0}\right)}{\phi_{0}}, T^{\prime}=\frac{T}{\Delta T}, C^{\prime}=\frac{C}{\Delta C}, H^{\prime}=\frac{H}{H}$.

Equations (1) - (8), in non-dimensional form can be written as:

$\nabla \cdot q^{\prime}=0$

$$
\begin{aligned}
\frac{1}{\operatorname{Pr}} \frac{\partial q^{\prime}}{\partial t}=-\nabla^{\prime} p^{\prime} & +\nabla^{2} q^{\prime}-R m \hat{e}_{z}+R a T^{\prime} \hat{e}_{z}-\frac{R s}{L e} C^{\prime} \hat{e}_{z} \\
& -R n \phi^{\prime} \hat{e}_{z}+\frac{P r}{P r_{M}} Q\left(H^{\prime} \cdot \nabla^{\prime}\right) H
\end{aligned}
$$

$\frac{\partial T^{\prime}}{\partial t^{\prime}}+q^{\prime} \cdot \nabla^{\prime} T^{\prime}=\nabla^{2} T^{\prime}+\frac{N_{B}}{L n} \nabla^{\prime} \phi^{\prime} \cdot \nabla T^{\prime}+\frac{N_{A} N_{B}}{L n} \nabla^{\prime} T^{\prime}$

$\frac{\partial C^{\prime}}{\partial t^{\prime}}+q^{\prime} \cdot \nabla C^{\prime}=\frac{1}{L e} \nabla^{2} C^{\prime}+S r \nabla^{2} T^{\prime}$

$\frac{\partial \phi^{\prime}}{\partial t^{\prime}}+q^{\prime} \cdot \nabla \phi^{\prime}=\frac{1}{L n} \nabla^{2} \phi^{\prime}+\frac{N_{A}}{L n} \nabla^{2} T^{\prime}$ 
$\frac{d H^{\prime}}{d t^{\prime}}=\left(H^{\prime} \cdot \nabla^{\prime}\right) q^{\prime}+\frac{P r}{P r_{M}} \nabla^{\prime 2} H^{\prime}$

$\nabla^{\prime} \cdot H^{\prime}=0$

where non-dimensional parameters are: $\operatorname{Ln}=\frac{\kappa}{D_{B}}$ is a nanofluid Lewis number, $L e=\frac{\kappa}{\kappa^{\prime}}$ Lewis number, $R a=\frac{\rho_{0} q \alpha d^{3} \Delta T}{\mu \kappa}$ is the thermal Rayleigh number, $R s=\frac{\rho_{0} g \alpha d^{3} \Delta C}{\mu \kappa}$ is the solutal Rayleigh number, $R m=\frac{\left(\rho_{p} \phi_{0}+\rho\left(1-\phi_{0}\right)\right) g d^{3}}{\mu \kappa}$ is the basic-density Rayleigh number, $\operatorname{Pr}=\frac{\mu}{\rho_{0} \kappa}$ is the Prandtl number, $P r_{M}=\frac{\mu}{\rho_{0} \eta}$ is the magnetic Prandtl number, $\mathrm{Q}=\frac{\mu_{e} H^{2} d^{2}}{4 \pi \rho_{0} v \eta}$ is the Chandrasekhar number, $R n=\frac{\left(\rho_{p}-\rho_{0}\right) \phi_{0} g d^{3}}{\mu \kappa}$ is the nanoparticle Rayleigh-Darcy number, $S r=\frac{D_{C T} \Delta T}{\kappa \Delta C}$ is the Soret parameter, $N_{A}=\frac{D_{T} \Delta T}{D_{B} T_{1} \phi_{0}}$ is the modified diffusivity ratio, $N_{B}=\frac{(\rho c)_{p} \phi_{0}}{\rho c}$ is the modified particle-density increment.

The dimensionless boundary conditions are:

$w=0, T=1, C=1, \frac{\partial \varphi}{\partial z}+N_{A} \frac{\partial T}{\partial z}=0$ at $z=0$

$w=0, T=0, C=0, \frac{\partial \varphi}{\partial z}+N_{A} \frac{\partial T}{\partial z}=0$ at $z=1$

\subsection{Basic Solutions}

The basic state of the nanofluid is assumed to be time independent and is described by $q^{\prime}(u, v, w)=0, p^{\prime}=p^{\prime}(z)$, $H^{\prime}=H_{b}(z), T^{\prime}=T_{b}(z), C=C_{b}(z), \phi=\phi_{b}(z)$.

The steady state solution is obtained as:

$T_{b}=1-z, C_{b}=1-z, \phi_{b}=\varphi_{0}+N_{A} Z$

where $\varphi_{0}$ is reference value for nanoparticles volume fraction.

The basic solution for temperature is same as the solution obtained by Chand (2013) while basic solution for the nanoparticles volume fraction is changed in comparison with Chand (2013).

But these basic solutions are identical with solutions obtained by Nield and Kuznetsov (2014).

\subsection{Perturbation Solutions}

To study the stability of the system, we superimposed infinitesimal perturbations on the basic state, which are of the forms:

$q^{\prime}=0+q^{\prime \prime}, T^{\prime}=T_{b,}+T^{\prime \prime}, \phi^{\prime}=\phi_{b}+\phi^{\prime \prime}, p^{\prime}$

$=p_{b}+p^{\prime \prime}, H^{\prime}=H_{b}+h\left(h_{x}, h_{y}, h_{z}\right)$

with $T_{b}=1-z, C_{b}=1-z, \phi_{b}=\phi_{0}+N_{A} z$

There after dropping the dashes ( ") for simplicity.

Using the equation (18) in the equations (9) - (15), we obtain the linearized perturbation (neglecting the product of the prime quantities) equations as:

$\nabla \cdot q=0$

$$
\begin{aligned}
\frac{1}{\operatorname{Pr}} \frac{\partial q}{\partial t}=-\nabla p+ & \nabla^{2} q+\operatorname{RaT} \hat{e}_{z}-\frac{R s}{L e} C \hat{e}_{z}-R n \phi \hat{e}_{z} \\
& +\frac{P r}{P r_{M}} Q \frac{\partial h}{\partial z}
\end{aligned}
$$

$\frac{\partial T}{\partial t}-w=\nabla^{2} T+\frac{N_{B}}{L n}\left(\frac{\partial T}{\partial z}-\frac{\partial \phi}{\partial z}\right)-\frac{2 N_{A} N_{B}}{\operatorname{Ln}} \frac{\partial T}{\partial z}$

$\frac{\partial C}{\partial t}-w=\frac{1}{L e} \nabla^{2} C+S r \nabla^{2} T$

$\frac{\partial \phi}{\partial t}+w=\frac{1}{L n} \nabla^{2} \phi+\frac{N_{A}}{L n} \nabla^{2} T$

$\frac{\partial h}{\partial t}=\frac{\partial w}{\partial z}+\frac{P r}{P r_{M}} \nabla^{2} h$

$\nabla \cdot h=0$

The boundary conditions are:

$w=0, T=0, C=0, \frac{\partial \varphi}{\partial z}+N_{A} \frac{\partial T}{\partial z}=0 a t z=0,1$

Now eliminating $p$ and $h$ from equations (20) by making use of equations (19) and (25), we get:

$\left(\left(\frac{P r}{P r_{M}} \nabla^{2}-\frac{\partial}{\partial t}\right)\left(\frac{1}{P r} \frac{\partial}{\partial t} \nabla^{2}-\nabla^{4}\right)+\frac{P r}{P r_{M}} Q D^{2} \nabla^{2}\right) w$
$=\left(\frac{P r}{P r_{M}} \nabla^{2}-\frac{\partial}{\partial t}\right)\left(R a \nabla_{H}^{2} T-\frac{R s}{L e} \nabla_{H}^{2} C-R n \nabla_{H}^{2} \phi\right)$

\section{NORMAL MODES ANALYSIS}

Analyzing the disturbances into the normal modes and assuming that the perturbed quantities are of the form:

$$
\begin{gathered}
{[w, T, \phi, C]=[W(z), \Theta(z), \Phi(z), \Gamma(z)] \exp \left(i k_{x} x\right.} \\
\left.+i k_{y} y+n t\right)
\end{gathered}
$$

where: $k_{x}, k_{y}$ are wave numbers in $x$ and $y$ direction and $n$ is growth rate of disturbances.

Using equation (28), equations (27), (19), (21) - (23) become:

$$
\begin{aligned}
\left(\left(\frac{P r}{P r_{M}}\left(D^{2}-a^{2}\right)\right.\right. & -n)\left(\frac{n}{P r}\left(D^{2}-a^{2}\right)-\left(D^{2}-a^{2}\right)^{2}\right) \\
& \left.+\frac{P r}{P r_{M}} Q D^{2}\left(D^{2}-a^{2}\right)\right) W \\
& +\left(\frac{P r}{P r_{M}}\left(D^{2}-a^{2}\right)-n\right)\left(a^{2} \operatorname{Ra\Theta }\right. \\
& \left.-a^{2} \frac{R s}{L e} \Gamma-a^{2} R n \Phi\right)=0
\end{aligned}
$$

$W+\left(D^{2}-a^{2}+\frac{N_{B}}{L n} D-\frac{2 N_{A} N_{B}}{L n} D-n\right) \Theta-\frac{N_{B}}{L n} D \Phi$
$=0$

$W+\operatorname{Sr}\left(D^{2}-a^{2}\right) \Theta+\left(\frac{1}{L e}\left(D^{2}-a^{2}\right)-n\right) \Gamma=0$

$W-\frac{N_{A}}{L n}\left(D^{2}-a^{2}\right) \Theta-\left(\frac{1}{L n}\left(D^{2}-a^{2}\right)-n\right) \Phi=0$

where $: D \equiv \frac{d}{d z}$ and $a=\sqrt{k_{x}^{2}+k_{y}^{2}}$ is dimensionless resultant wave number. 
The boundary conditions of the problem in view of normal mode analysis are:

$W=0, D^{2} W=\Theta=0, \Gamma=0, D \Phi+N_{A} D \Theta=0$

at $z=0,1$.

For neutral stability the real part of the $\mathrm{n}$ is zero. Hence now we writen $=i \omega$, (where $\omega$ is real and is dimensionless frequency of the oscillation).

\section{METHOD OF SOLUTION}

The Galerkin weighted residuals method is used to obtain an approximate solution to the system of equations (29) - (32) with the corresponding boundary conditions (33). On choosing trial functions (satisfying boundary condition (33)) $W, \Theta, \Gamma$ and $\Phi$ as:

$\mathrm{W}=\sum_{p=1}^{N} A_{p} \mathrm{~W}_{\mathrm{p}}, \Theta=\sum_{p=1}^{N} B_{p} \Theta_{\mathrm{p}}$

$\Gamma=\sum_{p=1}^{N} C_{p} \Gamma_{\mathrm{p}}, \Phi=\sum_{p=1}^{N} D_{p} \Phi_{\mathrm{p}}$

where: $A_{p}, B_{p}, C_{p}$ and $D_{p}$ are unknown coefficients, $p=1,2,3, \ldots N$ and the base functions $W_{p}, \Theta_{p}, \Gamma_{p}$ and $\Phi_{p}$ are assumed in the following form:

$W_{p}=z^{p+1}-2 z^{p+2}+z^{p+3}, \Theta_{p}=\Gamma_{p}=z^{p}-$

$z^{p+1} \Phi_{p}=-N_{A}\left(z^{p}-z^{p+1}\right)$

such that $W_{p}, \Theta_{p}, \Gamma_{p}$ and $\Phi_{p}$ satisfy the corresponding boundary conditions. Using expression for $W, \Theta, \Gamma$ and $\Phi$ in equations (29) - (32) and multiplying first equation by $W_{p}$ second equation by $\Theta_{p}$ and third by $\Gamma_{p}$ and forth by $\Phi_{p}$, and integrating in the limits from zero to unity, we obtain a set of $4 \mathrm{~N}$ linear homogeneous equations in $4 \mathrm{~N}$ unknown $A_{p}, B_{p}, C_{p}$ and $D_{p} ; p=1,2,3, \ldots N$. For existing of nontrivial solution, the vanishing of the determinant of coefficients produces the characteristics equation of the system in term of Rayleigh number $R a$. Thus $R a$ is found in terms of the other parameters.

\section{STATIONARY CONVECTION}

Oscillatory convection is ruled out because of the absence of the two opposing buoyancy forces so we consider the case of the stationary convection.

For the first Galerkin approximation we take $N=1$; the appropriate trial function for boundary condition (33) is given by:

$W_{p}=z^{2}(1-z)^{2}, \Theta_{p}=\Gamma_{p}=z(1-z), \Phi_{p}$

$$
=-N_{A} z(1-z)
$$

Substituting trail functions (36) in the system of equations (29) - (32) and using boundary condition (33), we obtain the eigenvalue equation as:

$\mathrm{R} a-R s(1-\mathrm{Sr})+\left(N_{A}+L n\right) L e R n$

$=\frac{28}{27 a^{2}}\left(a^{4}+24 a^{2}+504+12 Q\right)\left(a^{2}+10\right)$
In the absence of solute gradient $(R s=0, L e=1)$, the corresponding Rayleigh number $\mathrm{Ra}$ for steady onset is given byR $a=$ $\frac{28}{27 a^{2}}\left(a^{4}+24 a^{2}+504+12 Q\right)\left(a^{2}+10\right)-\left(N_{A}+L n\right) R n$. This is good agreement of the result obtained by Chand (2013).

In the absence of magnetic field $(Q=0)$, equation (37) reduces to:

$\mathrm{R} a-R s(1-S r)+\left(N_{A}+L n\right) L e R n$

$=\frac{28}{27 a^{2}}\left(a^{4}+24 a^{2}+504+12 Q\right)\left(a^{2}+10\right)$

The right- hand side of equation (38) takes the minimum value when $a=3.12$ and its minimum value is 1750 . Hence the onset stationary convection is given by: $\mathrm{R} a-R s(1-S r)+$ $\left(N_{A}+\operatorname{Ln}\right) \operatorname{LeRn}=1750$.

The value 1750 obtained using the Galerkin first term approximation is about 3\% greater than exact value 1707.76 for the critical Rayleigh number for the classical Rayleigh- Bénard problem.

In the absence of both magnetic field $(Q=0)$ and solute gradient $(R s=0, L e=1)$, the onset of stationary convection is given by $R a+\left(N_{A}+L n\right) R n=1750$.

This is good agreement of the result obtained by Nield and Kuznetsov (2014).

\section{RESULT AND DISCUSSION}

Double diffusive convection in a horizontal layer of nanofluid in the presence of vertical magnetic field with Soret effect is investigated. Equation (37) expresses the thermal stationary Rayleigh number $R a$ as a function of dimensionless wave number $a$ and magnetic field (Chandrasekhar number $Q$ ), Lewis number $L e$, nanofluid Lewis number $L n$, modified diffusivity ratio $N_{A}$, and nanoparticles Rayleigh number $R n$. It is also noted that parameter NB does not appear in the equation, thus instability is purely phenomenon due to buoyancy coupled with the conservation of nanoparticles. It is independent of the contributions of Brownian motion and thermophoresis to the thermal energy equation. The parameter $N_{B}$ drops out because of an orthogonal property of the first order trail functions and their first derivatives.

Now we discuss the results graphically. Numerical computations are carried out for different values of Chandrasekhar number $Q$, Lewis number $L e$, nanofluid Lewis number $L n$, Soret parameter $\mathrm{Sr}$ and solutal Rayleigh numberRs. The parameters considered are in the range of Chand and Rana $(2013,2014 a) 10^{2} \leq$ $R a \leq 10^{5}$ (thermal Rayleigh number), $10^{2} \leq R s \leq 10^{5}$ (solutal Rayleigh number), $10 \leq Q \leq 10^{3}$ (Chandrasekhar number), $1<\varepsilon<10^{-1}$ (porosity parameter), $0 \leq S r \leq 1$ (Soret parameter), $10^{-1} \leq$ Le $\leq 10$ (Lewis number) $10^{2} \leq \operatorname{Ln} \leq 10^{4}$ (nanofluid Lewis number), $1<N_{A}<10$ (modified diffusivity ratio), $1 \leq R n \leq 10$ (nanoparticle Rayleigh number).

The variation of the stationary thermal Rayleigh number as functions of the wave number for different sets of values for the different parameters are shown in Figs. $2-8$.

The stationary convection curves in $(R a, a)$ plane for various values of Chandrasekhar number $Q$ and fixed values of other parameters is shown in Fig. 2. It is found that the Rayleigh number increases with increase in the value of Chandrasekhar number $Q$, thus magnetic field has stabilizing effect on fluid layer. 


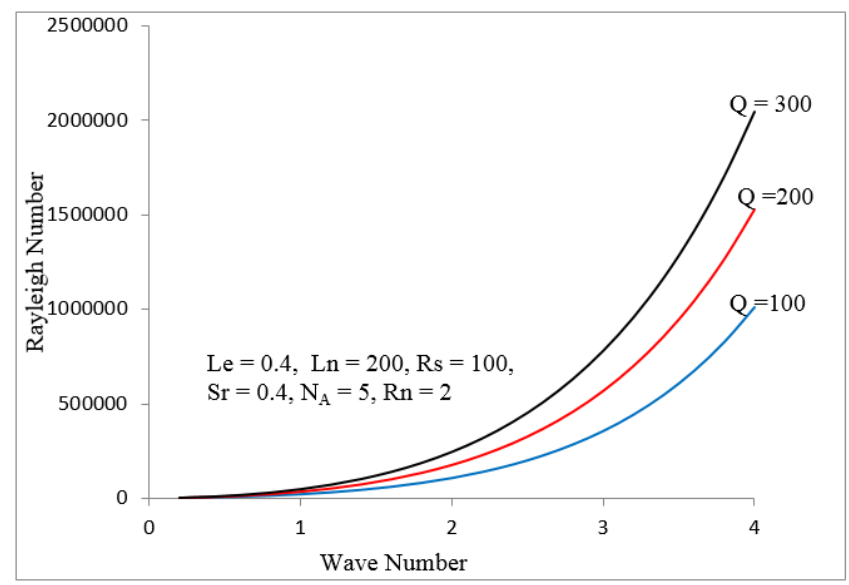

Fig. 2. Variation of Rayleigh number with wave number for different values of Chandrasekhar number

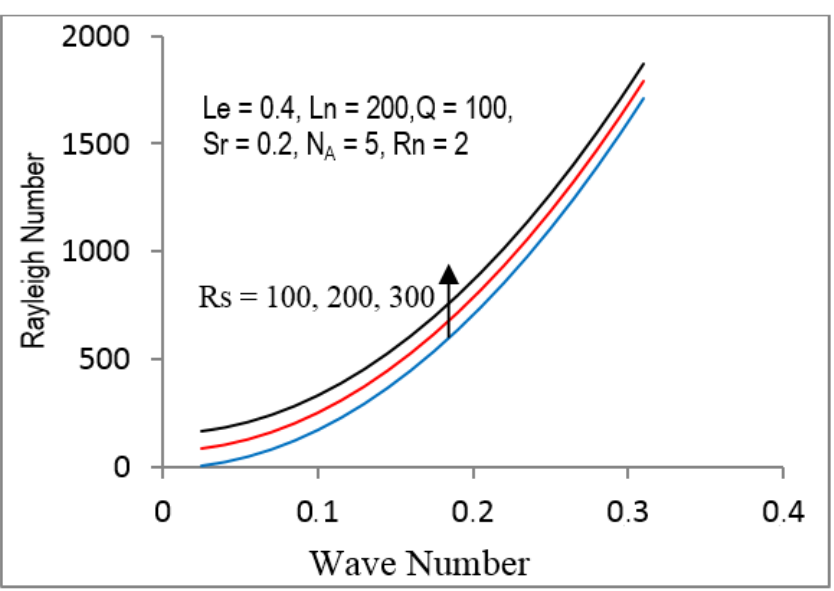

Fig. 3.Variation of stationary Rayleigh number with wave number for different values of solutal Rayleigh number

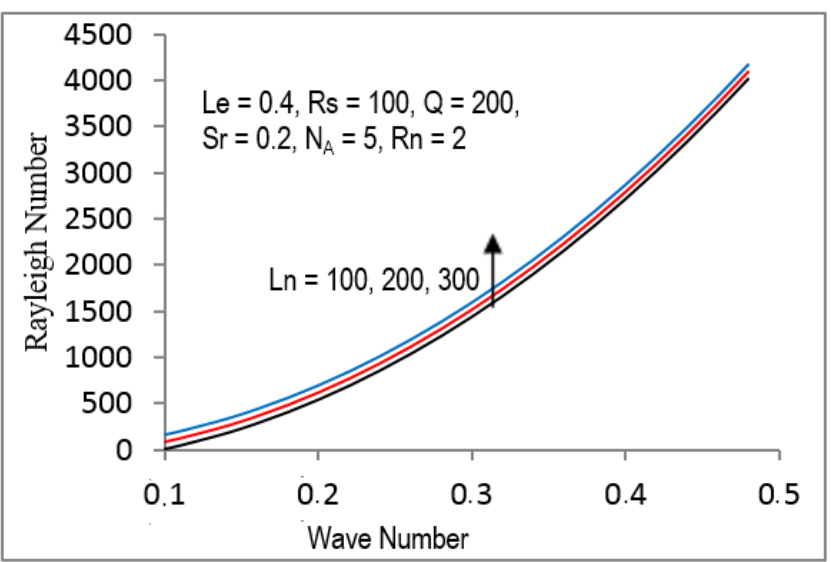

Fig. 4. Variation of stationary Rayleigh number with wave number for different values of nanofluid Lewis number

Fig. 3 shows the variation of thermal Rayleigh number with wave number for different values of solutal Rayleigh number. It is found that the thermal Rayleigh number increases as values of solutal Rayleigh number increases. Thus solutal Rayleigh number stabilizes the fluid layer.

Fig. 4 shows the variation of thermal Rayleigh number with wave number for different values of nanofluid Lewis number. It is found that the thermal Rayliegh number increases as values of nanofluid Lewis number increases. Thus nanofluid Lewis number stabilizes the fluid layer.
Fig. 5 shows the variation of Rayleigh number with wave number for different values of Soret parameter. It is found that the Rayleigh number decreases as values of Soret parameter increases. Thus Soret parameter has destabilizing effect on fluid layer.

Fig. 6 shows the variation of Rayleigh number with wave number for different values of nanoparticle Rayleigh number. It is found that the Rayleigh number decreases as values of nanoparticle Rayleigh number increases. Thus nanoparticle Rayleigh number destabilizes the fluid layer.

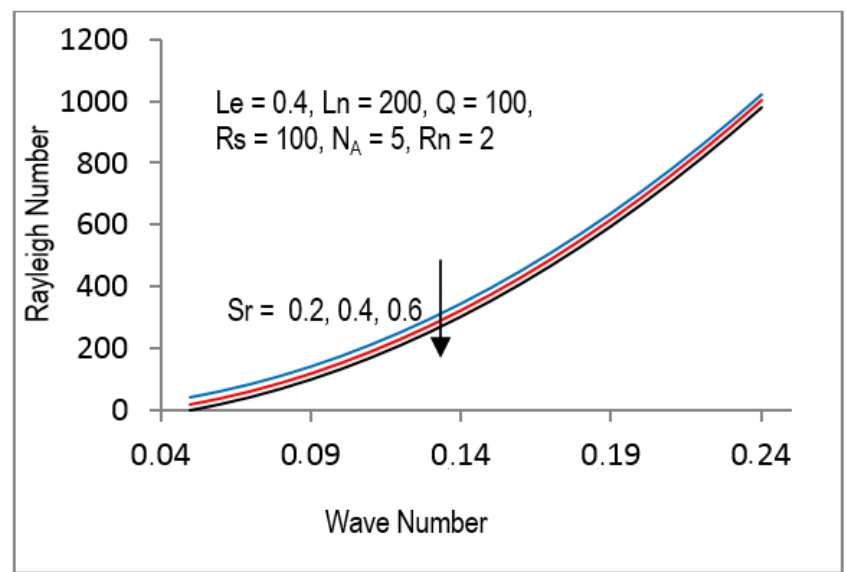

Fig. 5. Variation of stationary Rayleigh number with wave number for different values of Soret parameter

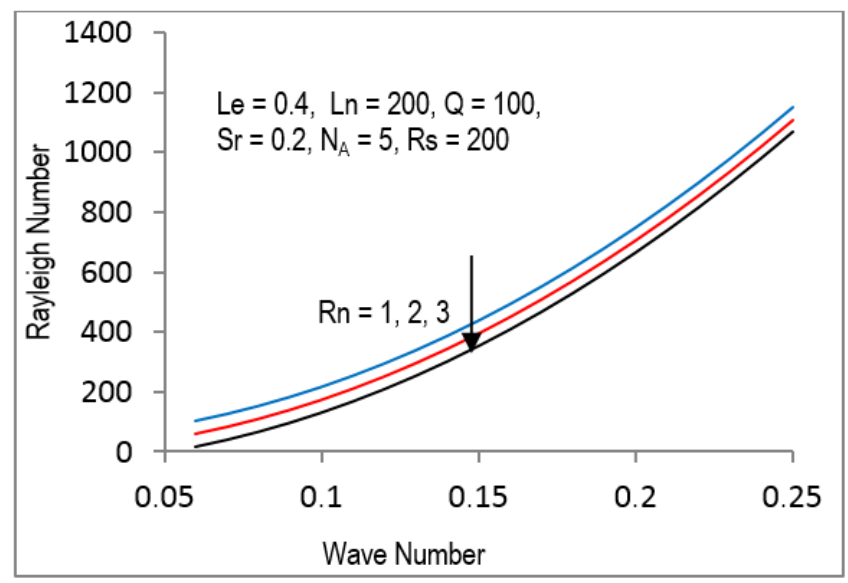

Fig. 6. Variation of stationary Rayleigh number with wave number for different values of nanoparticle Rayleigh number

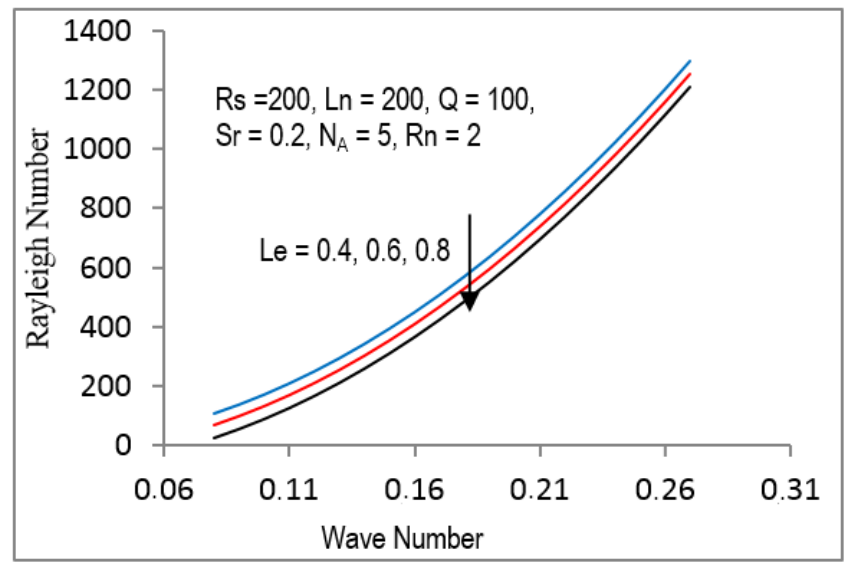

Fig. 7. Variation of stationary Rayleigh number with wave number for different values of Lewis number 


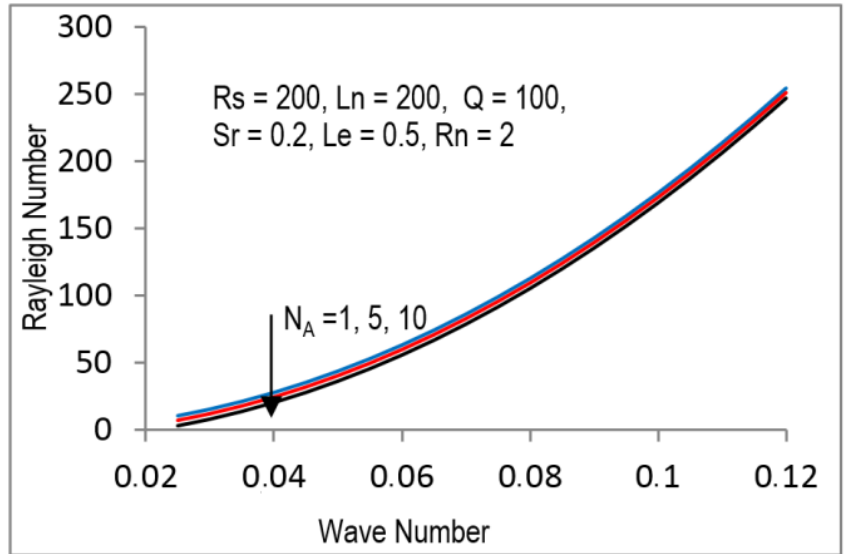

Fig. 8. Variation of Rayleigh number with wave number for different values of modified diffusivity ratio

Fig. 7 shows the variation of Rayleigh number with wave number for different values of Lewis number. It is found that the Rayleigh number decreases as values of Lewis number increases. Thus Lewis number destabilizes the fluid layer.

Fig. 8 shows the variation of Rayleigh number with wave number for different values of modified diffusivity ratio. It is found that the Rayleigh number decreases as values of modified diffusivity ratio increases. Thus modified diffusivity ratio has destabilizing effect on the fluid layer.

\section{CONCLUSIONS}

Double-diffusive convection in a horizontal layer of nanofluid in the presence of magnetic field with Soret effect is investigated for more realistic boundary conditions. The problem is analyzed boundaries which are isothermal and the flux of volume fractions of nanoparticles is zero on the boundaries. The resulting eigenvalue problem is solved numerically using the Galerkin technique.

The main conclusions are:

- The critical cell size is not a function of any thermo physical properties of nanofluid.

- Instability is purely phenomenon due to buoyancy coupled with the conservation of nanoparticles. It is independent of the contributions of Brownian motion and thermophoresis.

- Oscillatory convection is ruled out because of the absence of the two opposing buoyancy forces.

- For stationary convection the magnetic field, nanofluid Lewis number and solutal Rayleigh number stabilizes fluid layer, while Soret effect, Lewis number modified diffusivity ratio and nanoparticle Rayleigh number destabilize the fluid layer.

Nomenclature: $a$ - wave number, $C$ - solute concentration, $c_{p}$ - heat capacity, $d$ - thickness of fluid layer, $D_{B}$ - diffusion coefficient, $D_{T}$ - thermophoretic diffusion coefficient, $D_{C T}$-Soret coefficient, $g$ - acceleration due to gravity, $H$ - magnetic field, $k_{m}$ - thermal conductivity, $L e$ - Lewis number, $L n$ - nanofluid Lewis number, $n$ - growth rate of disturbances, $N_{A}$ - modified diffusivity ratio, $N_{B}$ - modified particle-density increment, $p$ - pressure, $P r$ - Prandtl number, $P r_{M}$ - magnetic Prandtl number, $q$ - velocity of fluid, $Q$ - Chandrasekhar number, $R a$-thermal Rayleigh number, $R a_{c}$ - critical Rayleigh number, $R m$ - density Rayleigh number, $R n$ - concentration Rayleigh number, $R s$ - solutal Rayleigh number, $t$ - time, $T$ - temperature, $T_{1}$ - reference scale for temperature, $(u, v, w)$ - components of fluid velocity, $(x, y, z)$
- space co-ordinates.

Greek symbols: $\alpha$ - thermal expansion coefficient, $\alpha^{\prime}$ - analogous solvent coefficient of expansion, $\mu_{e}$ - magnetic permeability, $\mu$ - viscosity, $\rho$ - density of the nanofluid, $\rho_{0}$ - density of the nanofluid at lower boundary, $\rho_{c}$ - heat capacity, $(\rho c)_{p}$ - heat capacity of the nanoparticles, $\varphi$ - volume fraction of the nanoparticles, $\varphi_{0}$-reference scale for nanoparticles fraction, $\rho_{p}$ - density of the nanoparticles, $\rho_{f}$ - density of base fluid, $\kappa$ - thermal diffusivity, $\kappa^{\prime}$ - solute diffusivity, $\omega$ - frequency of oscillation, $\partial$ - curly operator.

Superscripts: ' - non-dimensional variables, ' ' - perturbed quantities. Subscripts: $p$ - particle, $f$ - fluid, $s$ - stationary convection.

\section{REFERENCES}

1. Alchaar S., Vesseur P., Bilgen E.(1995), Effect of a magnetic field on the onset of convection in a porous medium, Heat and Mass Transfers, 30, 259-267.

2. Alloui Z., Vasseur P., Reggio M.(2010), Natural convection of nanofluids in a shallow cavity heated from below, International Journal of Thermal Science, 50(3), 385-393.

3. Bahloul A., Boutana N., Vasseur P. (2003), Double-diffusive and Soret-induced convection in a shallow horizontal porous layer, J. Fluid Mech., 491, 325-352.

4. Buongiorno J.(2006), Convective Transport in Nanofluids, ASMEJournal of Heat Transfer, 12, 240-250.

5. Chand R.(2013), On the onset of Rayleigh-Bénard convection in a layer of nanofluid in Hydromagnetics, Int. J. of Nanoscience, 12(6), 1350038-7.

6. Chand R., Kango S. K., Rana G. C. (2014),Thermal Instability in Anisotropic Porous Medium Saturated by a Nanofluid-A Realistic Approach, NSNTAIJ, 8(12), 445-453.

7. Chand R., Kango S. K., Singh V. (2015a), Megneto-convection in a layer of Maxwell visco-elastic fluid in a porous medium with Soret effect, Research J. of Engineering and Tech., 6(7), 23-30.

8. Chand R., Rana G. C. (2012a), Dufour and Soret effects on the thermosolutal instability of Rivlin-Ericksen elastico-viscous fluid in porous medium, Z. Naturforsch, 67a, 685-691.

9. Chand R., Rana G. C. (2012b), Oscillating convection of nanofluid in porous medium, Transp Porous Med., 95, 269-284.

10. Chand R., Rana G. C. (2012c), On the onset of thermal convection in rotating nanofluid layer saturating a Darcy-Brinkman porous medium, Int. J. of Heat and Mass Transfer, 55, 5417-5424.

11. Chand R., Rana G. C.(2012d), Thermal instability of Rivlin-Ericksen elastico-viscous nanofluid saturated by a porous medium, J. Fluids Eng., 134(12), 21203-7.

12. Chand R., Rana G. C.(2014a), Double diffusive convection in a layer of Maxwell visco-elastic fluid in porous medium in the presence of Soret and Dufour effects, Journal of Fluids, 2014, 1-7.

13. Chand R., Rana G. C. (2014b), Hall Effect on the thermal instability in a horizontal layer of nanofluid, Journal of Nanofluids, 3, 247-253.

14. Chand R., Rana G. C. (2014c), Thermal instability in a Brinkman porous medium saturated by nanofluid with no nanoparticle flux on boundaries, Special Topics \& Reviews in Porous Media: An International Journal, 5(4), 277-286.

15. Chand R., Rana G. C. (2015), Magneto convection in a layer of nanofluid in porous medium-A more realistic approach, Journal of Nanofluids, 4, 196-202.

16. Chand R., Rana G. C., Hussein A. K. (2015b), On the onset of thermal instability in a low Prandtl number nanofluid layer in a porous medium, Journal of Applied Fluid Mechanics, 8(2), 265-272.

17. Chandrasekhar S.(1961), Hydrodynamic and Hydromagnetic Stability, Oxford University Press, Dover Publication, New York.

18. Choi S.(1995), Enhancing Thermal Conductivity of Fluids with Nanoparticles in: D.A. Siginer and H. P. Wang (Eds), Developments and Applications of Non-Newtonian Flows, ASMEFED, Vol. 231/MDVol. 66, 99-105. 
19. Gupta U., Ahuja J., Wanchoo R. K. (2013), Magneto-convection in a nanofluid layer, Int. J. Heat and Mass Transfer, 64, 1163-1171.

20. Kim J.,Kang Y.T.,Choi C.K. (2011),Analysis of convective instability and heat transfer characteristics of nanofluids, Physics of Fluid, 16(7), 2395-2401.

21. Knobloch E., (1980), Convection in binary fluids, Phys. Fluids, 23(9), 1918-1920.

22. Kuznetsov A. V., Nield D. A. (2010a), Effect of local thermal nonequilibrium on the onset of convection in a porous medium layer saturated by a nanofluid, Transport in Porous Media, 83, 425-436.

23. Kuznetsov A. V., Nield D. A. (2010b), The onset of double-diffusive nanofluid convection in a layer of a saturated porous medium, Transport in Porous Media, 85(3),941-952.

24. Kuznetsov A. V., Nield D. A.(2011), Thermal instability in a porous medium layer saturated by a nanofluid: Brinkman Model, Transp. Porous Medium, 81(3), 409-422.

25. Motsa S. S. (2008), On the onset of convection in a porous layer in the presence of Dufour and Soret effects, SJPAM, 3, 58-65.

26. Nield D. A. (1968), Onset of thermohaline convection in a porous medium, Water Resour. Res., 4, 553-560.

27. Nield D. A., Kuznetsov A. V. (2009), Thermal instability in a porous medium layer saturated by a nanofluid, Int. J. Heat Mass Transf., 52, 5796-5801.

28. Nield D. A., Kuznetsov A. V. (2010a), The on set of convection in a horizontal nanofluid layer of finite depth, European Journal of Mechanics B/Fluids, 2, 217-223.

29. Nield D. A., Kuznetsov A. V. (2010b), The effect of local thermal non-equilibrium on the onset of convection in a nanofluid, J. Heat Transfer, 132(5),052405-052411.

30. Nield D. A., Kuznetsov A. V. (2010b), The onset of double-diffusive convection in a nanofluid layer, Int. J. of Heat and Fluid Flow, 32(4), $771-776$
31. Nield D. A., Kuznetsov A. V. (2011a), The onset of convection in a layer of cellular porous material: Effect of temperature-dependent conductivity arising from radiative transfer, J. Heat Transfer, 132(7), 074503-4.

32. Nield D. A., Kuznetsov A. V. (2014), Thermal instability in a porous medium layer saturated by a nanofluid: A revised model, Int. J. of Heat and Mass Transfer, 4, 68, 211-214.

33. Nield D. A., Manole D. M., Lage J. L. (1993), Convection induced by inclined thermal and thermosolutal gradients in a shallow horizontal layer of porous medium, J. Fluid Mech., 257, 559-568.

34. Patil R. P., Rudraiah N. (1973), Stability of hydromagnetic thermoconvective flow through porous medium, Transactions of the ASME Journal of Applied Mechanics, 40(E), 879-884.

35. Rudraiah N., Shrimani P. K., Friedrich R. (1982), Finite amplitude convection in two component fluid saturated porous layer, Int. J. Heat and MassTransfer, 25, 715-722.

36. Tzou D. Y. (2008a), Thermal in stability of nanofluids in natural convection, International Journal of Heat and Mass Transfer, 51, 2967-2979.

37. Tzou D. Y. (2008b), Instability of nanofluids in natural convection, ASME Journal of Heat Transfer, 130, 1-9.

38. Yadav D., Bhargava R., Agrawal G. S. (2013), Thermal instability in a nanofluid layer with a vertical magnetic field, J. Eng. Math., 80, 147-164.

The authors are grateful to the reviewers for their valuable comments and suggestions for improvement of the paper. 